\title{
UNIFORM ESTIMATES FOR THE PARABOLIC GINZBURG-LANDAU EQUATION
}

\author{
F. Bethuel ${ }^{1}$ And G. Orlandi ${ }^{2}$
}

\begin{abstract}
We consider complex-valued solutions $u_{\varepsilon}$ of the Ginzburg-Landau equation on a smooth bounded simply connected domain $\Omega$ of $\mathbb{R}^{N}, N \geq 2$, where $\varepsilon>0$ is a small parameter. We assume that the Ginzburg-Landau energy $E_{\varepsilon}\left(u_{\varepsilon}\right)$ verifies the bound (natural in the context) $E_{\varepsilon}\left(u_{\varepsilon}\right) \leq M_{0}|\log \varepsilon|$, where $M_{0}$ is some given constant. We also make several assumptions on the boundary data. An important step in the asymptotic analysis of $u_{\varepsilon}$, as $\varepsilon \rightarrow 0$, is to establish uniform $L^{p}$ bounds for the gradient, for some $p>1$. We review some recent techniques developed in the elliptic case in [7], discuss some variants, and extend the methods to the associated parabolic equation.
\end{abstract}

Mathematics Subject Classification. 35K55, 35J60, 58E50, 49J10.

Received December 17, 2001. Revised January 25, 2002.

\section{INTRODUCTION}

In many problems involving a small parameter $\varepsilon$ (tending to zero), a crucial step in order to describe the asymptotic limit of the solutions is to establish uniform estimates, i.e. independent of $\varepsilon$. Of course, when the limit is singular these estimates may involve a function space which is larger than the energy space. A typical example is the Cahn-Hilliard (also called Modica-Mortola) functional, where the energy space is $H^{1}$, whereas the minimizers happen to be uniformly bounded in $B V$ (see [29,30]).

In this paper we will focus on uniform estimates for the complex-valued Ginzburg-Landau equation. Here again the energy space is $H^{1}$; however uniform estimates are established (in the elliptic case) in $W^{1, p}$ with $1 \leq p<\frac{N}{N-1} \leq 2$ (see $\left.[7,9,11,26]\right)$. Our purpose is to review some new ideas introduced in [7] for the elliptic case, and then to extend these methods to the associated parabolic evolution problem.

More precisely, the following situation was analysed in [7]. Let $N$ be an integer larger than two, and let $\Omega$ be a smooth bounded, simply connected domain in $\mathbb{R}^{N}$. For $0<\varepsilon<1$ a small parameter, consider solutions $u_{\varepsilon}: \Omega \rightarrow \mathbb{C}$ of the Ginzburg-Landau equation with Dirichlet data $g_{\varepsilon}$ in $H^{1 / 2}(\partial \Omega ; \mathbb{C}):$

$$
\left\{\begin{aligned}
-\Delta u_{\varepsilon} & =\frac{1}{\varepsilon^{2}} u_{\varepsilon}\left(1-\left|u_{\varepsilon}\right|^{2}\right) & & \text { in } \Omega \\
u_{\varepsilon} & =g_{\varepsilon} & & \text { on } \partial \Omega
\end{aligned}\right.
$$

Keywords and phrases: Ginzburg-Landau, parabolic equations, Hodge-de Rham decomposition, Jacobians.

1 Analyse Numerique, Université P. et M. Curie, BC 187, 4 place Jussieu 75252 Paris Cedex 05, France;

e-mail: bethuel@ann.jussieu.fr

2 Dipartimento di Informatica, Università di Verona, strada le Grazie, 37134 Verona, Italy. 
Assume moreover that there exist positive constants $M_{0}$ and $M_{1}$ such that

$$
\begin{aligned}
& E_{\varepsilon}\left(u_{\varepsilon}\right)=\int_{\Omega} e_{\varepsilon}\left(u_{\varepsilon}\right)=\frac{1}{2} \int_{\Omega}\left|\nabla u_{\varepsilon}\right|^{2}+\frac{1}{4 \varepsilon^{2}} \int_{\Omega}\left(1-\left|u_{\varepsilon}\right|^{2}\right)^{2} \leq M_{0}|\log \varepsilon|, \\
& \left\|g_{\varepsilon}\right\|_{H^{1 / 2}(\partial \Omega)}^{2} \leq M_{1} \\
& \left|g_{\varepsilon}\right|=1, \quad \text { a.e. in } \partial \Omega \text {. }
\end{aligned}
$$

The main result of [7] is the following:

Theorem 1 ([7], Th. 1). Let $1 \leq p<\frac{N}{N-1}$. There exists a constant $C_{p}$ depending only on $M_{0}, M_{1}, \Omega$ and $p$, but independent of $\varepsilon$, such that for any solution $u_{\varepsilon}$ of $(G L)_{\varepsilon}$ verifying $(H 1),(H 2)$ and $(H 3)$, we have

$$
\int_{\Omega}\left|\nabla u_{\varepsilon}\right|^{p} \leq C_{p}
$$

The proof of Theorem 1 relies on a new result in [22] (see also [1]). Roughly speaking, this result shows that if, for $0<\varepsilon<1, v_{\varepsilon}: \Omega \rightarrow \mathbb{C}$ verifies the bound

$$
E_{\varepsilon}\left(v_{\varepsilon}\right) \leq M_{0}|\log \varepsilon|
$$

then the Jacobians $\left\{J v_{\varepsilon}\right\}_{0<\varepsilon<1}$ are precompact in some weak norm (see Sect. 2 for a precise statement), whereas the family $\left\{v_{\varepsilon}\right\}_{0<\varepsilon<1}$ may not be compact in any reasonable norm. The Jacobian $J v$ (for a map $v: \Omega \rightarrow \mathbb{C}$ ) is defined as

$$
J v:=\frac{1}{2} d(v \times d v)=\sum_{i<j}\left(v_{x_{i}} \times v_{x_{j}}\right) d x_{i} \wedge d x_{j}
$$

(here $a \times b:=a_{1} b_{2}-a_{2} b_{1}$ denotes the exterior product of two vectors $a, b \in \mathbb{R}^{2} \simeq \mathbb{C}$ ). Moreover, if $v_{\varepsilon_{n}}\left(\varepsilon_{n} \rightarrow 0\right.$ ) is a subsequence such that $J v_{\varepsilon_{n}}$ converges, then the limit $J_{*}$ is a measure with the structure of an integer multiplicity rectifiable current of dimension $N-2$.

In many cases (and here specially in view of the parabolic equation considered later) it is natural to relax the condition $\left|g_{\varepsilon}\right|=1$. Therefore, we will consider also the following variant of assumption (H3), namely we may assume instead that there exists a positive constant $M_{2}$ such that

$$
\frac{1}{2} \int_{\partial \Omega}\left|\nabla g_{\varepsilon}\right|^{2}+\frac{1}{4 \varepsilon^{2}} \int_{\partial \Omega}\left(1-\left|g_{\varepsilon}\right|^{2}\right)^{2} \leq M_{2}|\log \varepsilon| .
$$

We then have the following:

Theorem 1bis. Let $1 \leq p<\frac{N}{N-1}$. There exists a constant $C_{p}$ depending only on $M_{0}, M_{1}, M_{2}, \Omega$ and $p$, but independent of $\varepsilon$, such that for any solution $u_{\varepsilon}$ of $(G L)_{\varepsilon}$ verifying $(H 1),($ H2), (H3bis) we have

$$
\int_{\Omega}\left|\nabla u_{\varepsilon}\right|^{p} \leq C_{p}
$$

Estimate (1) was first considered in [9] for $N=2$, where it was established in the case $g_{\varepsilon}=g$ is independent on $\varepsilon$ and smooth (see also [6] for other references in case $N=2$ ). It was then generalized under various restrictive assumptions on $N, g_{\varepsilon}$ and $u_{\varepsilon}$ (see [11,26,31] and [14]). Theorem 1 and Theorem 1 bis cover all the above quoted results; however we expect that the same conclusion might be derived under milder assumptions on the boundary data $g_{\varepsilon}$.

As already mentioned, this kind of estimate is a crucial ingredient in the asymptotic analysis of solutions to equation $(\mathrm{GL})_{\varepsilon}$ as $\varepsilon \rightarrow 0$. The theory was developed during the last decade in $[3,6,8-11,14,26,27,31,33,36]$. In particular, the main result in [11] (Th. 1 there) can be derived under the assumptions considered here. More precisely, the following holds: 
Theorem 2. Let $u_{\varepsilon}$ be a solution of $\left(G L_{\varepsilon}\right)$ satisfying $(H 1),(H 2),(H 3)$ or $(H 3 b i s)$.

Then, for a subsequence $\varepsilon_{n} \rightarrow 0$, there exist a map $u_{*} \in W^{1, p}(\Omega), \forall 1 \leq p<\frac{N}{N-1}$, and a map $g_{*} \in H^{1 / 2}(\partial \Omega)$ such that

i) $\left|u_{*}\right|=1$ on $\Omega,\left|g_{*}\right|=1, u_{*}=g_{*}$ on $\partial \Omega$;

ii) $u_{\varepsilon_{n}} \rightarrow u_{*}$ in $W^{1, p}(\Omega), \quad g_{\varepsilon_{n}} \rightarrow g_{*}$ in $H^{1 / 2}(\partial \Omega)$;

iii) $\operatorname{div}\left(u_{*} \times \nabla u_{*}\right)=0 \quad$ in $\Omega$;

iv) $\frac{e_{\varepsilon_{n}}\left(u_{\varepsilon_{n}}\right)}{\left|\log \varepsilon_{n}\right|} \rightarrow \mu_{*}$ as measures, where $\mu_{*}$ is a bounded measure on $\bar{\Omega}$.

Set $\mathcal{S}=\operatorname{supp}\left(\mu_{*}\right)$.

v) $\mathcal{S}$ is a closed subset of $\bar{\Omega}$ with $\mathcal{H}^{\mathcal{N}-2}(\mathcal{S})<+\infty$;

vi) $u_{*} \in C^{\infty}(\Omega \backslash \mathcal{S})$ and for any ball $B\left(x_{0}, r\right)$ included in $\Omega \backslash \mathcal{S}$ there exists a function $\varphi_{*} \in C^{\infty}\left(B\left(x_{0}, r\right)\right)$, such that $\Delta \varphi_{*}=0, u_{*}=\exp \left(i \varphi_{*}\right)$;

vii) $u_{\varepsilon_{n}} \rightarrow u_{*}$ in $C^{k}(K)$, for any compact subset $K$ of $\Omega \backslash \mathcal{S}$;

viii) $\mathcal{S}$ is $\mathcal{H}^{N-2}$-rectifiable; $\mu_{*}$ is a stationary varifold.

The proof of Theorem 2 can be derived, following the same arguments as in Sections 4, 7, 8 and 9 of [11], from estimate (1) and the $\eta$-ellipticity property:

Theorem 3 ([11], Th. 2). Let $u_{\varepsilon}$ be a solution of $(G L)_{\varepsilon}$ on the ball $B_{r}$. Then there exist constants $K>0$, and $\alpha>0$, depending only on $N$ such that if

$$
E_{\varepsilon}\left(u_{\varepsilon}, B_{r}\right) \leq \eta r^{N-2}\left|\log \frac{\varepsilon}{r}\right|
$$

with $\eta>0$, then

$$
\left|u_{\varepsilon}(0)\right| \geq 1-K \eta^{\alpha}
$$

Note that in [11], estimate (1) appeared as a consequence of Theorem 3 (together with covering arguments), whereas here the two properties happen to be completely independent results. In particular, for the proof of Theorem 2, this approach bypasses the (somewhat unpleasant) technicalities related to the analysis near the boundary.

We recall also that statement viii) in Theorem 2 is a direct consequence of Theorem 3 and the analysis of [4] (rectifiability of $\mathcal{S}$ can be also deduced, as in [27], using the result of [25]).

In two dimensions, Theorem 3 originated simultaneously in $[12,36]$, and was used extensively for a large number of problems (see $[6,33]$ ). In higher dimension, the first $\eta$-ellipticity result was given in [31] under the name $\eta$-compactness (for $N=3$ and minimizing maps), then in [26] for minimizing maps in arbitrary dimension, in [27] for $N=3, u_{\varepsilon}$ not necessarily minimizing, and finally in $[10,11]$ in the general case.

Remark 1. From Theorem 2 we deduce directly that

$$
J u_{\varepsilon_{n}} \rightarrow J_{*}=J u_{*} \quad \text { in } \mathcal{D}^{\prime}(\Omega) .
$$

It can be proved directly arguing as in Sections 5 and 6 of [11] (without the machinery of $[1,22]$ ), that $J_{*}$ is a bounded measure and that

$$
\operatorname{supp}\left(J_{*}\right) \subset \mathcal{S}=\operatorname{supp}\left(\mu_{*}\right) .
$$

When $g_{\varepsilon}$ varies with $\varepsilon$, then the two sets might be different. However, if $g_{\varepsilon} \equiv g$ is fixed (and $\left.|g|=1\right)$, then it is not known if the two sets coincide; it is even not known if the rectifiable set supporting $J_{*}$ is closed or not. Finally, we have (see Rem. 5.1)

$$
u_{*} \in C^{\infty}\left(\Omega \backslash \operatorname{supp}\left(J_{*}\right)\right)
$$


We turn next to the parabolic Ginzburg-Landau equation, which is the main focus of this paper:

$(\mathrm{PGL})_{\varepsilon}$

$$
\begin{cases}\frac{\partial u_{\varepsilon}}{\partial t}-\Delta u_{\varepsilon}=\frac{1}{\varepsilon^{2}} u_{\varepsilon}\left(1-\left|u_{\varepsilon}\right|^{2}\right) & \text { in } \Omega \times(0,+\infty) \\ u_{\varepsilon}(x, 0)=u_{\varepsilon}^{0}(x) & \text { for a.e. } x \in \Omega, \\ u_{\varepsilon}(x, t)=g_{\varepsilon}(x) & \text { for a.e. }(x, t) \in \partial \Omega \times(0,+\infty) .\end{cases}
$$

Equations related to $(\mathrm{PGL})_{\varepsilon}$ appear in many applications, for instance as dynamical models in superconductivity. The equation $(\mathrm{PGL})_{\varepsilon}$ has been extensively studied in recent years. In particular the dynamics of vortices has been described in the two dimensional case, and some results have been obtained in higher dimensions (see e.g. $[5,20,21,23,24])$.

In this paper, we will make several assumptions on the initial data $u_{\varepsilon}^{0}: \Omega \rightarrow \mathbb{C}$ and on the boundary condition $g_{\varepsilon}: \partial \Omega \rightarrow \mathbb{C}$, which is time independent. First, for $g_{\varepsilon}$, we assume (H2), (H3) or (H3bis) hold, as in the elliptic case. For $u_{\varepsilon}^{0}$ we assume that there exist positive constants $M_{0}$ and $M_{3}$ such that

$$
E_{\varepsilon}\left(u_{\varepsilon}^{0}\right) \leq M_{0}|\log \varepsilon|, \quad\left\|u_{\varepsilon}^{0}\right\|_{H^{1 / 2}(\Omega)}^{2} \leq M_{3}, \quad\left|u_{\varepsilon}^{0}\right| \leq 1 .
$$

Under these hypotheses, equation $(\mathrm{PGL})_{\varepsilon}$ admits, for fixed $\varepsilon>0$, a unique solution $u_{\varepsilon}: \Omega \times[0,+\infty) \rightarrow \mathbb{C}$. Moreover, $u_{\varepsilon} \in C^{\infty}(\Omega \times(0, T))$ for each $T>0$, and $\left|u_{\varepsilon}\right| \leq 1$, by the maximum principle. For $t \geq 0$, let $u_{\varepsilon}^{t}: \Omega \rightarrow \mathbb{C}$ be the function defined by $u_{\varepsilon}^{t}(x):=u_{\varepsilon}(x, t)$, and recall the energy equality

$$
E_{\varepsilon}\left(u_{\varepsilon}^{T}\right)+\int_{0}^{T} \int_{\Omega}\left|\frac{\partial u_{\varepsilon}}{\partial t}\right|^{2}=E_{\varepsilon}\left(u_{\varepsilon}^{0}\right) .
$$

In particular we have the inequality

$$
\int_{0}^{T} \int_{\Omega}\left|\frac{\partial u_{\varepsilon}}{\partial t}\right|^{2}+\left|\nabla u_{\varepsilon}\right|^{2} \leq M_{0}(T+1)|\log \varepsilon|
$$

and hence $u_{\varepsilon} \in H^{1}(\Omega \times[0, T])$, and the $L^{2}$ norm of its gradient (with respect to space-time variables) is bounded by a constant times $|\log \varepsilon|$, as in the elliptic case.

Our purpose is to extend some of the techniques developed in the elliptic framework: in particular, Hodge-de Rham decomposition, reduction to systems of linear equations, etc. One of the consequences of this analysis is the following estimate, which, to our knowledge, is new.

Theorem 4. Let $1 \leq p<\frac{N+1}{N}, T>0$. There exists a constant $C_{p}$ depending only on $M_{0}, M_{1}, M_{2}, M_{3}, \Omega, p$ and $T$, but independent of $\varepsilon$, such that for any solution $u_{\varepsilon}$ of $(P G L)_{\varepsilon}$ with initial data $u_{\varepsilon}^{0}$ verifying (H4) and boundary data $g_{\varepsilon}$ verifying (H2), and (H3) or (H3bis), we have

$$
\int_{0}^{T} \int_{\Omega}\left|\nabla u_{\varepsilon}\right|^{p} \leq C_{p}
$$

A direct consequence of Theorem 4 and the analysis in Section 6 is the following:

Proposition 1. Let $\left\{u_{\varepsilon}\right\}_{0<\varepsilon<1}$ be solutions of $(P G L)_{\varepsilon}$ satisfying (H4), (H2), and (H3) or (H3bis). Then, for a subsequence $\varepsilon_{n} \rightarrow 0$, there exists a map $u_{*}: \Omega \times[0,+\infty) \rightarrow \mathbb{C}$ such that, for every $1 \leq p<\frac{N+1}{N}$ and every $T>0$,

i) $u_{\varepsilon_{n}} \rightarrow u_{*}$ in $L^{p}(\Omega \times[0, T]), \nabla u_{\varepsilon_{n}} \rightarrow \nabla u_{*}$ in $L^{p}(\Omega \times[0, T])$; 
ii) $u_{*} \in C^{\infty}\left(\Omega \times[0,+\infty) \backslash \operatorname{supp}\left(\tilde{J}_{*}\right) ; S^{1}\right)$.

Here $\tilde{J}_{*}$ is the weak limit of $\tilde{J} u_{\varepsilon_{n}}$ in the sense of $[1,22]$ (see Sect. 2).

As one easily sees, Proposition 1 gives only partial results concerning the convergence of $u_{\varepsilon}$ as $\varepsilon$ goes to zero. Note in particular, that at this stage we cannot even exclude the fact that the support of $\tilde{J}_{*}$ has positive measure. A further step for the asymptotic analysis of $u_{\varepsilon}$ would be to derive the analogous of Theorem 3 for the parabolic case. In this context, a result in case $\Omega=\mathbb{R}^{3}$ is provided in [28].

The relation of the (possible) asymptotic behavior of solutions to (PGL) $)_{\varepsilon}$ with motion by mean curvature, in Brakke's (weak) formulation, has been shown in [4], under some additional assumption on the solutions, which is conjectured there. This assumption can be proved in the elliptic case, yielding, as already mentioned, statement viii) of Theorem 2 .

Finally, in the case the initial condition $u_{\varepsilon}^{0}$ has some special properties (in particular, the concentration set of $u_{\varepsilon}^{0}$ is a smooth $(N-2)$-dimensional manifold), then convergence to motion by mean curvature (for the concentration set), up to appearance of singularities, is established in [21] (see also [24]). The techniques there, rely on a careful analysis on the concentration of the energy density $e_{\varepsilon}\left(u_{\varepsilon}\right)$.

The outline of the paper is as follows.

The next section is devoted to an important estimate first derived by Jerrard and Soner [22] for Jacobians of maps $v_{\varepsilon}: \Omega \rightarrow \mathbb{C}$ verifying the bound $E_{\varepsilon}\left(v_{\varepsilon}\right) \leq M_{0}|\log \varepsilon|$. We present several variants of this estimate (most of the ideas are from [7]), which take into account the boundary data. These estimates are the main ingredient in the proofs of Theorem 1, Theorem 1bis and Theorem 4. Section 3 is concerned with the Hodge-de Rham decomposition of $u \times \nabla u$, and its interplay with the results of Section 2. Section 4 deals with a similar issue, for a situation specially adapted for the parabolic problem. In Section 5 we give the proofs of Theorem 1 and Theorem 1bis. Finally, in Section 6 we prove Theorem 4.

\section{UNIFORM BOUNDS FOR JACOBIANS}

In this section, we will describe and extend a new estimate for Jacobians of maps in $H^{1}$ with some control on the Ginzburg-Landau energy. This estimate is actually in the same spirit as, in the scalar case (i.e. for real-valued maps $v$ ), the famous estimate (see $[29,30]$ )

$$
\int_{\Omega}|\nabla \Phi(v)| \leq \sqrt{2} \varepsilon E_{\varepsilon}(v)
$$

where $\Phi(v)=\frac{v^{2}}{2}-\frac{v^{3}}{3}$ is a primitive of $\left(1-v^{2}\right)$. Estimate (2.1) is the starting point of the strong $L^{1}$ compactness of sequences of functions $v_{\varepsilon}$ satisfying $\varepsilon E_{\varepsilon}\left(v_{\varepsilon}\right) \leq C$ (and not only of solutions of $(\mathrm{G} L)_{\varepsilon} !$ ).

For complex-valued maps $v \in H^{1}(\Omega ; \mathbb{C})$ satisfying the even stronger bound (but natural in this context) $E_{\varepsilon}(v) \leq C|\log \varepsilon|$, we can not expect similar compactness properties. A simple example is provided by maps presenting wild oscillations in the phase, for instance take $v_{\varepsilon}=\exp \left(i|\log \varepsilon|^{1 / 2} \phi\right)$, where $\phi: \Omega \rightarrow \mathbb{R}$ is an arbitrary smooth function. Note that compactness cannot be expected even for solutions of (GL) $\varepsilon_{\varepsilon}$ (see [15]).

However, oscillations in the phase of $v$ are not seen by the Jacobian of $v$, which, we recall, is defined as the two-form

$$
J v:=\frac{1}{2} d(v \times d v)=\sum_{i<j}\left(v_{x_{i}} \times v_{x_{j}}\right) d x_{i} \wedge d x_{j}
$$

In particular, $v_{x_{i}} \times v_{x_{j}}=0$ whenever $v_{x_{i}}$ and $v_{x_{j}}$ are colinear. Hence, when $|v|=1$ as in the example above, we have $J v \equiv 0$.

It turns out that Jacobians possess compactness properties in some weak norm, as was first shown by Jerrard and Soner in [22]. More precisely, from the computations in [22] we deduce immediately: 
Theorem 2.1. Let $\alpha>0, M>0$, and $U$ be a smooth bounded domain in $\mathbb{R}^{N}$. There exists a constant $K_{0}>0$, depending only on $U, M$ and $\alpha$, but independent of $\varepsilon$, such that for every map $v_{\varepsilon} \in H^{1}(U, \mathbb{C})$ verifying the bound

$$
E_{\varepsilon}\left(v_{\varepsilon}, U\right)=\frac{1}{2} \int_{U}\left|\nabla v_{\varepsilon}\right|^{2}+\frac{1}{4 \varepsilon^{2}} \int_{U}\left(1-\left|v_{\varepsilon}\right|^{2}\right)^{2} \leq M|\log \varepsilon|
$$

we have

$$
\left\|J v_{\varepsilon}\right\|_{\left[C_{c}^{0, \alpha}(U)\right]^{*}} \leq K_{0}
$$

Remark 2.1. Theorem 2.1 extends immediately to the case $U$ is a compact manifold. Note that in case $U$ is a compact manifold without boundary, then $C_{c}^{0, \alpha}(U)=C^{0, \alpha}(U)$.

The proof of Theorem 2.1 relies on reduction to the two dimensional case by slicing arguments, and then on lower bounds established in [19] (see also [34]) which generalize earlier results in [9, 17].

A different proof, which works also for $\mathbb{R}^{k}$-valued maps, has been derived independently in [1] using Geometric Measure Theory: the strategy is to approximate the Jacobian of $v_{\varepsilon}$ by an integer multiplicity polyhedral current with uniformly bounded mass, and then apply Federer-Fleming compactness theorem (see $[16,35]$ ).

Finally, a totally different approach is provided in [2]: it relies on the parabolic regularization of $v_{\varepsilon}$, as in [3], and on the regularity theory of [11].

In order to prove Theorem 1 and Theorem 1bis, we will make use of the following variant of Theorem 2.1.

Proposition 2.1. Let $\alpha>0$ and let $\Omega$ be a smooth domain. Let $g_{\varepsilon}: \partial \Omega \rightarrow \mathbb{C}$ satisfying either

i) $(\mathrm{H} 2)$ and $(\mathrm{H} 3)$

or

ii) (H3bis).

Then there exists a constant $K_{1}>0$ depending on $\alpha$, either $M_{1}$ or $M_{2}, \Omega$, but independent of $\varepsilon$, such that for every map $v_{\varepsilon} \in H^{1}(\Omega, \mathbb{C})$ verifying (2.3) and $v_{\varepsilon}=g_{\varepsilon}$ on $\partial \Omega$ we have

$$
\left\|J v_{\varepsilon}\right\|_{\left[C^{0, \alpha}(\bar{\Omega})\right]^{*}} \leq K_{1}
$$

Remark that condition i) yields some compactness on $g_{\varepsilon}$, whereas this is not the case for condition ii). In case assumption i) holds, the proof of Proposition 2.1 was given in [7]. We will give here the proof under the assumption ii) (it is actually even simpler than under hypothesis i)), and then recall some elements of the proof in case $\mathrm{i}$ ).

The idea, in both cases, is to extend the map $v_{\varepsilon}$ to some larger domain $G$ containing $\Omega$, in such a way that the Ginzburg-Landau energy as well as the Jacobian of the extension remain controlled. Then we apply Theorem 2.1 to this particular extension of $v_{\varepsilon}$ on the larger domain $G$.

Proof of Proposition 2.1 assuming $i)$. For $x \in \mathbb{R}^{N}$ set $d(x):=\operatorname{dist}(x, \Omega)$. Consider, for $\delta>0$ the set $W_{\delta}=$ $\left\{x \in \mathbb{R}^{N} \backslash \bar{\Omega}, d(x)<\delta\right\}$. For $\delta_{0}>0$ sufficiently small, on $W_{0} \equiv W_{\delta_{0}}$ the nearest-point projection $\pi: W_{0} \rightarrow \partial \Omega$ is well-defined and smooth, and its restriction to each level set $d^{-1}(t)$ in $W_{0}$ gives rise to diffeomorphisms $\pi_{t}: d^{-1}(t) \rightarrow \partial \Omega$.

Remark 2.2. Recall that for each $x \in W_{0}, d$ is differentiable at $x$ and $|\nabla d(x)|=1$. Moreover, $\pi(x)=$ $x-d(x) \nabla d(x)$, hence for each level $t,\left\|\nabla \pi_{t}-I\right\|_{L^{\infty}\left(W_{0}\right)} \leq C t$, where the constant $C$ depends only on the curvature of $\partial \Omega$.

Set $G=W_{0} \cup \bar{\Omega}$. We extend $v_{\varepsilon}$ to a map $\tilde{v}_{\varepsilon}$ defined on $G$ by setting

$$
\begin{cases}\tilde{v}_{\varepsilon}(x)=v_{\varepsilon}(x) & \text { for } x \in \bar{\Omega} \\ \tilde{v}_{\varepsilon}(x)=g_{\varepsilon}(\pi(x)) & \text { for } x \in W_{0} .\end{cases}
$$


For the Ginzburg-Landau energy $E_{\varepsilon}\left(\tilde{v}_{\varepsilon}, G\right)$ of $\tilde{v}_{\varepsilon}$ on $G$ we have the straightforward estimate (by (H3bis) and Rem. 2.2)

$$
E_{\varepsilon}\left(\tilde{v}_{\varepsilon}, G\right)=E_{\varepsilon}\left(v_{\varepsilon}, \Omega\right)+E_{\varepsilon}\left(\tilde{v}_{\varepsilon}, W_{0}\right) \leq\left(M_{0}+C \delta_{0} M_{2}\right)|\log \varepsilon|
$$

with $C$ depending on the curvature of $\partial \Omega$.

We turn now to the Jacobian $J \tilde{v}_{\varepsilon}$. Observe first that $J \tilde{v}_{\varepsilon}=\pi_{t}^{*} J g_{\varepsilon}$ on each level set $d^{-1}(t) \subset W_{0}$, while obviously $J \tilde{v}_{\varepsilon}=J v_{\varepsilon}$ in $\Omega$.

Let $f:\left[0, \delta_{0}\right] \rightarrow[0,1]$ a smooth cut-off function such that $f(t)=1$ for $t<\delta_{0} / 3, f(t)=0$ for $t>2 \delta_{0} / 3$. We will extend any smooth 2-form $\zeta \in C^{\infty}\left(\bar{\Omega} ; \Lambda^{2} \mathbb{R}^{N}\right)$ to a (Lipschitz continuous) 2-form $\tilde{\zeta}$ compactly supported in $G$ as follows: on $\partial \Omega$ decompose $\zeta=\zeta_{T}+\zeta_{N}$ where $\zeta_{T}$ and $\zeta_{N}$ denote respectively the tangential and the normal part of $\zeta$ with respect to $\partial \Omega$ (see for instance the Appendix of [11] for notations). Then, for a fixed $0<t<\delta_{0}$, set

$$
\tilde{\zeta}(x)=f(t)\left[\left(\pi_{t}^{-1}\right)^{*} \zeta_{\top}(x)+\zeta_{N}(\pi(x))\right], \quad \text { for each } x \in d^{-1}(t)
$$

A simple calculation yields, for each $\alpha>0,\|\tilde{\zeta}\|_{C^{0, \alpha}(G)} \leq C\|\zeta\|_{C^{0, \alpha}(\bar{\Omega})}$, where $C$ depends only on $\|f\|_{C^{0, \alpha}}$ and the curvature of $\partial \Omega$.

In view of (2.6) and Theorem 2.1 we have

$$
\left|\int_{G} J \tilde{v}_{\varepsilon} \cdot \tilde{\zeta}\right| \leq\left\|J \tilde{v}_{\varepsilon}\right\|_{\left[C_{c}^{0, \alpha}(G)\right]^{*}}\|\tilde{\zeta}\|_{C^{0, \alpha}(G)} \leq C_{1}\|\zeta\|_{C^{0, \alpha}(\bar{\Omega})} .
$$

We compute, using the coarea formula and Remark 2.2 ,

$$
\begin{aligned}
\left|\int_{W_{0}} J \tilde{v}_{\varepsilon} \cdot \tilde{\zeta}\right| & =\left|\int_{W_{0}}\right| \nabla d\left|\pi^{*} J g_{\varepsilon} \cdot \tilde{\zeta}\right|=\left|\int_{0}^{\delta_{0}} \mathrm{~d} t \int_{d^{-1}(t)} f(t) \pi_{t}^{*} J g_{\varepsilon} \cdot\left(\pi_{t}^{-1}\right)^{*} \zeta_{\top}\right| \\
& \leq C \delta_{0}\left|\int_{\partial \Omega} J g_{\varepsilon} \cdot \zeta \top\right| \leq C \delta_{0}\left\|J g_{\varepsilon}\right\|_{\left[C^{0, \alpha}(\partial \Omega)\right]^{*}}\|\zeta\|_{C^{0, \alpha}(\partial \Omega)} \\
& \leq C_{2}\|\zeta\|_{C^{0, \alpha}(\bar{\Omega})}
\end{aligned}
$$

where the last inequality follows from assumption (H3bis) and Remark 2.1 in the case $U=\partial \Omega$. Combining (2.8) and (2.9) we finally deduce

$$
\left|\int_{\Omega} J v_{\varepsilon} \cdot \zeta\right| \leq\left|\int_{G} J \tilde{v}_{\varepsilon} \cdot \tilde{\zeta}\right|+\left|\int_{W_{0}} J \tilde{v}_{\varepsilon} \cdot \tilde{\zeta}\right| \leq\left(C_{1}+C_{2}\right)\|\zeta\|_{C^{0, \alpha}(\bar{\Omega})}
$$

and the conclusion follows.

Proof of Proposition 2.1 assuming ii). The argument is based also on an extension of $v_{\varepsilon}$ to a larger domain $G$. However, the construction is different and is more involved. It yields a control of the Jacobian of the extension in the (stronger) $L^{1}$ norm. It is based on the following lemma, proved in [7].

Lemma 2.1 ([7], Prop. 4). Let $U$ be a smooth bounded domain of $\mathbb{R}^{N}$. There exists a constant $K_{2}>0$ depending only on $U$ such that for every $\gamma \in H^{1 / 2}\left(\partial U, S^{1}\right)$ there exists $w_{\varepsilon} \in H^{1}\left(U, \mathbb{R}^{2}\right)$ verifying

$$
\begin{aligned}
w_{\varepsilon} & =\gamma \quad \text { on } \partial U \\
E_{\varepsilon}\left(w_{\varepsilon}, U\right) & \leq K_{2}\|\gamma\|_{H^{1 / 2}(\partial U)}^{2}|\log \varepsilon| \\
\left\|J w_{\varepsilon}\right\|_{L^{1}(U)} & \leq K_{2}\|\gamma\|_{H^{1 / 2}(\partial U)}^{2} .
\end{aligned}
$$


We recall that a map $w_{\varepsilon}$ verifying $(2.11,2.12)$ was already constructed in [13] (Th. 5). Using a projection argument of [18], a different construction was provided in [32]. The construction in [7] follows the ideas of [18,32].

With Lemma 2.1 at our disposal, the proof is completed as follows: define $G=W_{0} \cup \bar{\Omega}$ as in case i), and set $U=W_{0}$, so that $\partial U=\partial \Omega \cup \partial G$. Let $\gamma$ be the function defined on $\partial U$ by $\gamma \equiv g$ on $\partial \Omega$ and $\gamma \equiv 1$ on $\partial G$, so that $\|\gamma\|_{H^{1 / 2}(\partial U)} \leq C\|g\|_{H^{1 / 2}(\partial \Omega)}$. Let $w_{\varepsilon}$ be the function defined on $U$ as in Lemma 2.1, and set

$$
\begin{cases}\tilde{v}_{\varepsilon}(x)=v_{\varepsilon}(x) & \text { for } x \in \Omega \\ \tilde{v}_{\varepsilon}(x)=w_{\varepsilon}(x) & \text { for } x \in U\end{cases}
$$

In particular $\tilde{v}_{\varepsilon} \in H^{1}(G, \mathbb{C})$, and clearly

$$
E_{\varepsilon}\left(\tilde{v}_{\varepsilon}, G\right) \leq E_{\varepsilon}\left(v_{\varepsilon}, \Omega\right)+E_{\varepsilon}\left(w_{\varepsilon}, U\right) \leq C\left(M_{0}+M_{1}\right)|\log \varepsilon| .
$$

Let $\zeta$ and $\tilde{\zeta}$ be as in case i). In view of (2.14) and Theorem 2.1 we deduce

$$
\left|\int_{G} J \tilde{v}_{\varepsilon} \cdot \tilde{\zeta}\right| \leq K\|\tilde{\zeta}\|_{C_{c}^{0, \alpha}(G)} \leq C K\|\zeta\|_{C^{0, \alpha}(\bar{\Omega})}
$$

where $K$ depends on $\delta_{0}, M_{0}, M_{1}, \alpha$. On the other hand $\left\|J w_{\varepsilon}\right\|_{L^{1}(U)} \leq C\|g\|_{H^{1 / 2}}^{2} \leq C M_{1}$ by (2.13), so that

$$
\left|\int_{U} J w_{\varepsilon} \cdot \tilde{\zeta}\right| \leq C M_{1}\|\tilde{\zeta}\|_{L^{\infty}(U)} \leq C M_{1}\|\tilde{\zeta}\|_{C_{c}^{0, \alpha}(G)} \leq C K\|\zeta\|_{C^{0, \alpha}(\bar{\Omega})}
$$

and the conclusion follows arguing as for (2.10) using (2.15) and (2.16).

Next, we adapt the previous discussion to a situation which we will encounter in the parabolic case. For that purpose, let $T>0$ and set

$$
\Lambda_{T}=\Omega \times[0, T] \subset \mathbb{R}^{N+1}
$$

For $0 \leq t \leq T$, we consider the slices $\Omega_{t}=\Omega \times\{t\}$, so that

$$
\partial \Lambda_{T}=\partial \Omega \times[0, T] \cup \Omega_{0} \cup \Omega_{T} .
$$

In what follows, $\nabla v_{\varepsilon}$ denotes the gradient of $v_{\varepsilon}$ with respect to spatial variables, whereas $\tilde{\nabla} v_{\varepsilon}$ represents the gradient with respect to all space-time variables $\left(x^{1}, \ldots, x^{N}, t\right)$. Similarly, $J v_{\varepsilon}$ (resp. $\tilde{J} v_{\varepsilon}$ ) will denote the spatial component of the Jacobian (resp. the Jacobian with respect to all variables $\left.x^{1}, \ldots, x^{N}, t\right)$.

Let $g_{\varepsilon}: \partial \Omega \rightarrow \mathbb{C}$ be given. We consider functions $v_{\varepsilon}: \Lambda_{T} \rightarrow \mathbb{C}$ verifying

$$
v_{\varepsilon}(x, t)=g_{\varepsilon}(x) \quad \text { in } \partial \Omega \times[0, T] .
$$

We will assume that for some positive constant $M_{0}$

$$
\begin{aligned}
& \int_{\Omega_{t}}\left|\nabla v_{\varepsilon}\right|^{2}+\frac{1}{2 \varepsilon}\left(1-\left|v_{\varepsilon}\right|^{2}\right)^{2} \leq M_{0}|\log \varepsilon| \quad \text { for } t=0, T \\
& \int_{\Lambda_{T}}\left|\tilde{\nabla} v_{\varepsilon}\right|^{2}+\frac{1}{2 \varepsilon}\left(1-\left|v_{\varepsilon}\right|^{2}\right)^{2} \leq M_{0}(T+1)|\log \varepsilon|
\end{aligned}
$$

Proposition 2.2. Let $\alpha>0, T>0$, and $v_{\varepsilon}: \Lambda_{T} \rightarrow \mathbb{C}$. Assume that $v_{\varepsilon}$ verifies (2.17, 2.18) and (2.19). Assume moreover that $g_{\varepsilon}$ verifies either (H2) and (H3), or (H3bis). Then there exists a constant $K_{2}>0$ depending on $M_{0}$, either $M_{1}$ or $M_{2}, \alpha, \Omega$ and $T$ but independent on $\varepsilon$, such that

$$
\left\|\tilde{J} v_{\varepsilon}\right\|_{\left[C^{0, \alpha}\left(\bar{\Lambda}_{T}\right)\right]^{*}} \leq K_{2} .
$$


Proof. Let $W_{0}$ and $G$ be as in the proof of Proposition 2.1 and consider the domain $Q_{T}=G \times(-1, T+1)$, so that $\Lambda_{T} \subset \subset Q_{T}$. We construct an extension $\check{v}_{\varepsilon}$ of $v_{\varepsilon}$ to $Q_{T}$ setting,

$$
\begin{cases}\check{v}_{\varepsilon}(x, t)=\tilde{v}_{\varepsilon}^{t}(x), & \forall t \in[0, T], \forall x \in W_{0} \\ \check{v}_{\varepsilon}(x, t)=\tilde{v}_{\varepsilon}^{0}(x), & \forall t \in(-1,0), \forall x \in W_{0} \\ \check{v}_{\varepsilon}(x, t)=\tilde{v}_{\varepsilon}^{T}(x), & \forall t \in(T, T+1) \forall x \in W_{0}\end{cases}
$$

where, for a map $w: \Omega \rightarrow \mathbb{C}, \tilde{w}$ defines its extension to the domain $G$ as in the proof of Proposition 2.1 (the definition is different in case (H3) and in case (H3bis)). Similarly, for a test function $\zeta \in C^{0, \alpha}\left(\bar{\Lambda}_{T}, \Lambda^{2} \mathbb{R}^{N+1}\right)$, we define its extension $\check{\zeta}$ to the larger domain $Q_{T}$, by

$$
\begin{cases}\check{\zeta}(x, t)=\tilde{\zeta}^{t}(x) & \forall t \in[0, T], \forall x \in W_{0} \\ \check{\zeta}(x, t)=\chi(t) \tilde{\zeta}^{0}(x) & \forall t \in(-1,0), \forall x \in W_{0} \\ \check{\zeta}(x, t)=\chi(t) \tilde{\zeta}^{T}(x) & \forall t \in(T, T+1), \forall x \in W_{0}\end{cases}
$$

Here, for a test function $\psi \in C^{0, \alpha}\left(\bar{\Omega} ; \Lambda^{2} \mathbb{R}^{N+1}\right), \tilde{\psi}$ denotes its extension to the domain $G$ as in the proof of Proposition 2.1 (again, the definition is different in case (H3) and in case (H3bis)). The function $\chi: \mathbb{R} \rightarrow \mathbb{R}^{+}$ denotes a cut-off function such that $\chi(t)=0$ if $t \leq-1$ or $t \geq T+1$.

We then have

$$
E_{\varepsilon}\left(\check{v}_{\varepsilon}, Q_{T}\right) \leq C E_{\varepsilon}\left(v_{\varepsilon}, \Lambda_{T}\right)+E_{\varepsilon}\left(v_{\varepsilon}^{0}, \Omega\right)+E_{\varepsilon}\left(v_{\varepsilon}^{T}, \Omega\right) \leq C M_{0}(T+1)|\log \varepsilon| .
$$

Therefore, we may apply Theorem 2.1 to $\check{v}_{\varepsilon}$ on $Q_{T}$, to assert that

$$
\left|\int_{Q_{T}} J \check{v}_{\varepsilon} \cdot \check{\zeta}\right|=\left|\int_{\Lambda_{T}} J v_{\varepsilon} \cdot \zeta+\int_{Q_{T} \backslash \Lambda_{T}} J \check{v}_{\varepsilon} \cdot \check{\zeta}\right| \leq C|| \check{\zeta}\left\|_{C^{0, \alpha}\left(Q_{T}\right)} \leq C\right\| \zeta \|_{C^{0, \alpha}\left(\bar{\Lambda}_{T}\right)} .
$$

Arguing as in the proof of Proposition 2.1, we estimate the integral of $J \check{v}_{\varepsilon} \cdot \check{\zeta}$ on the three components of $Q_{T} \backslash \Lambda_{T}=W_{0} \times[0, T] \cup G \times(-1,0) \cup G \times(T, T+1)$, and complete the proof as above.

\section{HodGe-De Rham Decomposition}

\subsection{Splitting of the energy}

Let $x_{0} \in \Omega$, and assume that $v: \Omega \rightarrow \mathbb{C}$ is smooth, and $v\left(x_{0}\right) \neq 0$. Then $v \neq 0$ in some open neighborhood $U$ of $x_{0}$, so that we may write

$$
v(x)=\rho(x) \exp (i \phi(x)), \quad \text { for } x \in U,
$$

where $\rho=|v|$ and $\phi$ is a real-valued function on $U$, defined up to an integer multiple of $2 \pi$. Moreover,

$$
\nabla v=\exp (i \phi) \nabla \rho+i \rho \exp (i \phi) \nabla \phi
$$

and $|\nabla v|^{2}$ splits as

From (3.2) we also notice that

$$
|\nabla v|^{2}=|\nabla \rho|^{2}+\rho^{2}|\nabla \phi|^{2}
$$

$$
v \times \nabla v=\rho^{2} \nabla \phi .
$$


When $v$ vanishes somewhere in $\Omega$, we are not able to define the function $\phi$ to the whole of $\Omega$. However, $v \times \nabla v$ is globally well-defined on $\Omega$, and we have, as above, the identity

$$
|v|^{2}|\nabla v|^{2}=\left.|v|^{2}|\nabla| v\right|^{2}+|v \times \nabla v|^{2}
$$

and hence

$$
|\nabla v|^{2}=|\nabla| v||^{2}+|v \times \nabla v|^{2}+\left(1-|v|^{2}\right)\left(|\nabla v|^{2}-\left.|\nabla| v\right|^{2}\right)
$$

Since $|\nabla v|^{2} \geq|\nabla| v||^{2}$, this yields

$$
|\nabla v|^{2} \leq|\nabla| v||^{2}+|v \times \nabla v|^{2}+\left.\left.|1-| v\right|^{2}|| \nabla v\right|^{2}
$$

so that

$$
|\nabla v|^{2} \leq|\nabla| v||^{2}+|v \times \nabla v|^{2}+\sqrt{2} \varepsilon\left[\frac{\left(1-|v|^{2}\right)^{2}}{4 \varepsilon^{2}}+\frac{|\nabla v|^{2}}{2}\right]
$$

i.e.

$$
|\nabla v|^{2} \leq|\nabla| v||^{2}+|v \times \nabla v|^{2}+\sqrt{2} \varepsilon e_{\varepsilon}(v)
$$

In view of (3.3), for every $1 \leq p \leq 2$ there exists some constant $C_{p}$ depending on $p$ and $\Omega$ such that

$$
\int_{\Omega}|\nabla v|^{p} \leq C_{p}\left[\left.\int_{\Omega}|\nabla| v\right|^{p}+\int_{\Omega}|v \times \nabla v|^{p}+\left(\varepsilon E_{\varepsilon}(v)\right)^{p / 2}\right] .
$$

Remark 3.1. Assume $v$ verifies $E_{\varepsilon}(v) \leq M_{0}|\log \varepsilon|$. Then, for $0<\varepsilon<1$ we have $\varepsilon|\log \varepsilon| \leq 1$, and consequently

$$
\varepsilon E_{\varepsilon}(v) \leq M_{0}
$$

\subsection{Hodge-de Rham decomposition for $v \times \nabla v$}

The Hodge-de Rham decomposition asserts that every $l$-form $\mu$ on a simply connected domain $\Omega$ can be decomposed as

$$
\mu=d H+d^{*} \Phi
$$

where $H$ is a $(l-1)$-form on $\Omega, \Phi$ represents a $(l+1)$-form, $d$ represents the exterior derivative, and $d^{*}= \pm \star d \star$ (here $\star$ denotes the Hodge operator). In general there is no uniqueness of such a decomposition. We may therefore impose auxiliary conditions, in particular on the boundary. A common choice of auxiliary conditions is

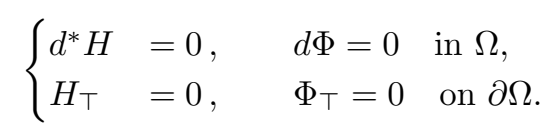

These conditions ensure uniqueness of the decomposition. Moreover, for any $1<p<+\infty$ there exists a constant $C_{p}$ depending on $p$ and $\Omega$, such that

$$
\|H\|_{W^{1, p}}+\|\Phi\|_{W^{1, p}} \leq C_{p}\|\mu\|_{L^{p}}
$$

Next let $v: \Omega \rightarrow \mathbb{C}$ be a function in $H^{1}$. We apply the previous decomposition to the 1 -form $v \times d v$, where $d v=\sum_{i=1}^{N} \partial_{i} v d x^{i}$. Therefore,

$$
v \times d v=d \varphi+d^{*} \psi
$$


where $\varphi$ is a real-valued function in $\Omega$ (and hence $d^{*} \varphi=0$ ) and $\psi$ is a 2 -form in $\Omega$, such that

$$
\begin{cases}d \psi=0 & \text { in } \Omega \\ \psi_{\top}=0 \quad \varphi=0 & \text { on } \partial \Omega\end{cases}
$$

Applying the $d$ operator to (3.6), we obtain

$$
2 J v=d(v \times d v)=d d^{*} \psi=d d^{*} \psi+d^{*} d \psi=-\Delta \psi \quad \text { in } \Omega .
$$

Hence $\psi$ is solution to the boundary problem

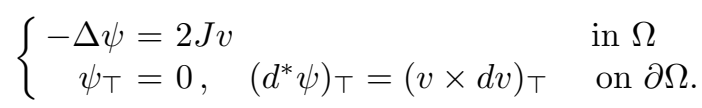

This elliptic problem determines the 2-form $\psi$ uniquely as a function of $J v$ and the boundary value of $v$ on $\partial \Omega$.

\section{3. $\boldsymbol{W}^{\mathbf{1}, p}$ estimates for $\psi$}

In this section we prove:

Proposition 3.1. Let $1 \leq p<\frac{N}{N-1}$, and assume $v \equiv v_{\varepsilon}: \Omega \rightarrow \mathbb{C}$ verifies (H1), (H2), and (H3) or (H3bis), and let $\psi_{\varepsilon} \equiv \psi$ be given by (3.6, 3.7). Then we have

$$
\int_{\Omega}\left|\nabla \psi_{\varepsilon}\right|^{p} \leq K_{p}
$$

where $K_{p}$ is a constant depending only on $p, M_{0}, M_{1}$ and $M_{2}$ in case (H3bis).

Remark 3.2. Here we do not assume that $v_{\varepsilon}$ is a solution of (GL) $)_{\varepsilon}$. Proposition 3.1 ensures compactness of the " $d^{*} \psi$ " component of $v \times d v$. This part accounts in particular for topological obstructions to the lifting property (3.1).

In order to prove Proposition 3.1, we need the following linear estimate related to (3.8) (this estimate is standard for functions).

Lemma 3.1. Let $1<p<+\infty$ and $\frac{1}{p}+\frac{1}{q}=1$. Let $l \in \mathbb{N}, 1 \leq l \leq N$. Let $\varphi$ and $\omega$ be $l$-forms on $\Omega$, and $A$ be an $(l-1)$-form on $\partial \Omega$. Assume that

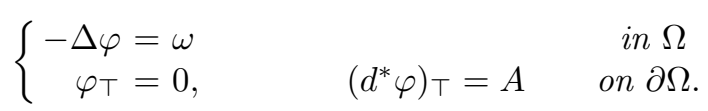

There exists some constant $C$ depending only on $\Omega$ and $p$ such that

$$
\|\varphi\|_{W^{1, p}(\Omega)} \leq C\left(\|\omega\|_{\left[W^{1, q}(\Omega)\right]^{*}}+\|A\|_{\left[W^{1-\frac{1}{q}, q}(\partial \Omega)\right]^{*}}\right) .
$$

We apply Lemma 3.1 to $\omega=2 J v$ and $A=(v \times d v)_{\top}=g_{\varepsilon} \times d g_{\varepsilon}$. Since $1 \leq p<\frac{N}{N-1}$, we have $q>N$, and, for $\alpha=1-\frac{N}{p}$, we recall the embedding $W^{1, q}(\Omega) \hookrightarrow C^{0, \alpha}(\bar{\Omega})$. By duality we therefore have the embedding

$$
\left[C^{0, \alpha}(\bar{\Omega})\right]^{*} \hookrightarrow\left[W^{1, q}(\Omega)\right]^{*}
$$


If $v_{\varepsilon}$ verifies (H1), (H2), (H3) or (H3bis), by Proposition 2.1 we have $\left\|J v_{\varepsilon}\right\|_{\left[C^{0, \alpha}(\bar{\Omega})\right]^{*}} \leq C$, hence by (3.9) we have

$$
\left\|J v_{\varepsilon}\right\|_{\left[W^{1, q}(\Omega)\right]^{*}} \leq C_{p}
$$

In order to apply Lemma 3.1 it remains to estimate $\left\|g_{\varepsilon} \times d g_{\varepsilon}\right\|_{\left[W^{1-\frac{1}{q}, q}(\partial \Omega)\right]^{*}}$.

Proposition 3.2. Let $q>N$ and $g \in H^{1 / 2}(\partial \Omega ; \mathbb{C})$ such that $\|g\|_{L^{\infty}(\partial \Omega)} \leq 1$. Then there is a constant $C_{q}>0$ depending only on $q$ and $\Omega$ such that

$$
\|g \times d g\|_{\left[W^{1-\frac{1}{q}, q}(\partial \Omega)\right]^{*}} \leq C_{q}\left(\|g\|_{H^{1 / 2}(\partial \Omega)}^{2}+\|g\|_{H^{1 / 2}(\partial \Omega e g a)}\right) .
$$

Proof. Let $\xi \in W^{1-\frac{1}{q}, q}(\partial \Omega)$. Note that we have the embedding $W^{1-\frac{1}{q}, q}(\partial \Omega) \hookrightarrow C^{0} \cap H^{1 / 2}(\partial \Omega)$. On the other hand, we recall that $H^{1 / 2} \cap L^{\infty}(\partial \Omega)$ is an algebra, therefore since $|g| \leq 1, \xi g$ belongs to $H^{1 / 2} \cap L^{\infty}(\partial \Omega)$ and

$$
\begin{aligned}
\|\xi g\|_{H^{1 / 2}(\partial \Omega)} & \leq C\left(\|\xi\|_{L^{\infty}}\|g\|_{H^{1 / 2}}+\|g\|_{L^{\infty}}\|\xi\|_{H^{1 / 2}}\right) \\
& \leq C\left(\|\xi\|_{W^{1-\frac{1}{q}, q}(\partial \Omega)}\|g\|_{H^{1 / 2}(\partial \Omega)}+\|\xi\|_{W^{1-\frac{1}{q}, q}(\partial \Omega)}\right) \\
& \leq\|\xi\|_{W^{1-\frac{1}{q}, q}(\partial \Omega)}\left(1+\|g\|_{H^{1 / 2}(\partial \Omega)}\right) .
\end{aligned}
$$

Finally, we have

$$
\begin{aligned}
\left|\int_{\partial \Omega} \xi(g \times d g)\right| & \leq\|d g\|_{H^{-1 / 2}(\partial \Omega)}\|\xi g\|_{H^{1 / 2}(\partial \Omega)} \\
& \leq C\|g\|_{H^{1 / 2}(\partial \Omega)}\|\xi\|_{W^{1-\frac{1}{q}, q}(\partial \Omega)}\left(1+\|g\|_{H^{1 / 2}(\partial \Omega)}\right),
\end{aligned}
$$

and the conclusion follows:

Proposition 3.2bis. Let $q>N$ and $g \in H^{1 / 2}(\partial \Omega ; \mathbb{C})$ such that

$$
\frac{1}{2} \int_{\partial \Omega}\left|\nabla g_{\varepsilon}\right|^{2}+\frac{1}{4 \varepsilon^{2}} \int_{\partial \Omega}\left(1-\left|g_{\varepsilon}\right|^{2}\right)^{2} \leq M_{2}|\log \varepsilon|
$$

Then for some constant $K_{q}>0$ depending only on $q, \Omega, M_{2}$ and $\|g\|_{H^{1 / 2}(\partial \Omega)}$, we have

$$
\|g \times d g\|_{\left[W^{1-\frac{1}{q}, q}(\partial \Omega)\right]^{*}} \leq K_{q} .
$$

Proof. Consider the function $\tilde{g}$ defined on $\partial \Omega$ by $\tilde{g}=g$ if $|g| \leq 1, \tilde{g}=g /|g|$ otherwise. We have $\tilde{g} \times d \tilde{g}=g \times d g$ if $|g| \leq 1$, and $\tilde{g} \times d \tilde{g}=\frac{1}{|g|^{2}} g \times d g$, if $|g| \geq 1$. Therefore

$$
|g \times d g-\tilde{g} \times d \tilde{g}| \leq\left.|| g\right|^{2}-1|| d g \mid \leq \sqrt{2} \varepsilon\left(\frac{\left(1-\left|g_{\varepsilon}\right|^{2}\right)^{2}}{4 \varepsilon^{2}}+\frac{|\nabla g|^{2}}{2}\right) .
$$

Let $\xi$ be as in Proposition 3.2. We have, as in (3.11),

$$
\left|\int_{\partial \Omega} \xi(\tilde{g} \times d \tilde{g})\right| \leq C\|\xi\|_{W^{1-\frac{1}{q}, q}(\partial \Omega)}\left(\|g\|_{H^{1 / 2}(\partial \Omega)}^{2}+\|g\|_{H^{1 / 2}(\partial \Omega)}\right) .
$$


On the other hand, in view of (3.12), we deduce

$$
\left|\int_{\partial \Omega} \xi(\tilde{g} \times d \tilde{g}-g \times d g)\right| \leq C\|\xi\|_{W^{1-\frac{1}{q}, q}(\partial \Omega)} M_{2} \varepsilon|\log \varepsilon| .
$$

Combining (3.13) with (3.14) we are led to

$$
\left|\int_{\partial \Omega} \xi(g \times d g)\right| \leq C\|\xi\|_{W^{1-\frac{1}{q}, q}(\partial \Omega)}\left(\|g\|_{H^{1 / 2}(\partial \Omega)}^{2}+\|g\|_{H^{1 / 2}(\partial \Omega)}+M_{2} \varepsilon|\log \varepsilon|\right),
$$

and the conclusion follows.

Proof of Proposition 3.1. In view of Lemma 3.1 and (3.8), we have

$$
\left\|\psi_{\varepsilon}\right\|_{W^{1, p}(\Omega)} \leq C\left(\left\|J v_{\varepsilon}\right\|_{\left[W^{1, q}(\Omega)\right]^{*}}+\left\|g_{\varepsilon} \times d g_{\varepsilon}\right\|_{W^{1-\frac{1}{q}, q}(\partial \Omega)}\right),
$$

where $q$ is such that $\frac{1}{p}+\frac{1}{q}=1$. The conclusion follows from (3.10), and Proposition 3.2 (in case (H3bis), from Prop. 3.2bis).

\section{Hodge-De Rham decomposition on $\Lambda_{T}$}

We will consider here a situation specially tailored for the parabolic case.

As in Section 2 consider, for $T>0$, the cylinder $\Lambda_{T}=\Omega \times[0, T] \subset \mathbb{R}^{N+1}$. Let $g_{\varepsilon}$ satisfy (H2), and (H3) or (H3bis). We will consider maps $v_{\varepsilon}: \Lambda_{T} \rightarrow \mathbb{C}$; recall that for $0 \leq t \leq T$, we have defined

$$
v_{\varepsilon}^{t}: \Omega \rightarrow \mathbb{C}, \quad v_{\varepsilon}^{t}(x)=v_{\varepsilon}(x, t)
$$

We will assume throughout this section that $v_{\varepsilon}$ verifies $(2.17-2.19)$, and

$$
\begin{aligned}
\left|v_{\varepsilon}\right| & \leq 1 \\
\left\|v_{\varepsilon}^{0}\right\|_{H^{1 / 2}(\Omega)} & \leq M_{3} .
\end{aligned}
$$

We also recall that $\tilde{\nabla}$ represents the gradient in $\mathbb{R}^{N+1}$, and we denote by $\delta$ the exterior derivative in $\mathbb{R}^{N+1}$, and $\delta^{*}= \pm \star \delta \star$, where $\star$ is the Hodge operator on $\mathbb{R}^{N+1}$.

Proposition 4.1. Let $v_{\varepsilon}$ be as above. Then there exist a function $\Phi$, a 1-form $\chi$ and a 2 -form $\Psi$ on $\Lambda_{T}$, such that

$$
\left\{\begin{array}{l}
v_{\varepsilon} \times \delta v_{\varepsilon}=\delta \Phi+\delta^{*} \Psi+\chi \\
\delta \Psi=0 \quad \text { in } \Lambda_{T} \\
\Phi=0 \quad \text { on } \Omega_{0} \cup \partial \Omega \times[0, T], \quad \Psi_{\top}=0 \quad \text { on } \partial \Lambda_{T}
\end{array}\right.
$$

Moreover, for $1 \leq p<\frac{N+1}{N}$, there exist constants $C_{p}$ and $0<\alpha<1$, depending on $p, T, \Omega$, such that

$$
\|\tilde{\nabla} \Psi\|_{L^{p}\left(\Lambda_{T}\right)} \leq C_{p}, \quad\|\chi\|_{L^{p}\left(\Lambda_{T}\right)} \leq C_{p} \varepsilon^{\alpha}
$$

Comment. Although the statement of Proposition 4.1 looks very similar to that of Proposition 3.1, we have to point out a major difference: on $\Omega_{T} \subset \partial \Lambda_{T}$ no uniform bound (and hence no compactness) is assumed for $v_{\varepsilon}$. In particular, this is the reason why we do not impose $\Phi=0$ on $\Omega_{T}$. 
Proof of Proposition 4.1. We decompose the proof into two steps. The first step corresponds to a Hodge-de Rham decomposition of $v_{\varepsilon}$ on $\Omega_{T} \subset \partial \Lambda_{T}$ : this allows to analyse the possible lack of compactness on this portion of $\partial \Lambda_{T}$. In the second step, we use a gauge transformation to remove this possible lack of compactness.

Step 1: HdR decomposition on $\Omega_{T}$. In view of the results of Section 3, we write

$$
v_{\varepsilon}^{T} \times d v_{\varepsilon}^{T}=d \varphi^{T}+d^{*} \psi^{T} \quad \text { in } \Omega,
$$

where $\varphi^{T}$ (resp. $\psi^{T}$ ) is a function (resp. a 2-form) defined on $\Omega$, satisfying

$$
d \psi^{T}=0 \quad \text { in } \Omega, \quad \varphi^{T}=0 \text { and } \psi_{\top}^{T}=0 \quad \text { on } \partial \Omega .
$$

By Proposition 3.1 and assumption (2.19), we have, for $1 \leq p<\frac{N}{N-1}$,

$$
\left\|\nabla \psi^{T}\right\|_{L^{p}(\Omega)}^{p} \leq C_{p}, \quad\left\|\nabla \psi^{T}\right\|_{L^{2}(\Omega)}^{2}+\left\|\nabla \varphi^{T}\right\|_{L^{2}(\Omega)}^{2} \leq C M_{0}|\log \varepsilon|
$$

We consider the harmonic extension $\Phi_{0}$ of $\varphi^{T}$ to $\Lambda_{T}$ defined by

$$
\begin{cases}\tilde{\Delta} \Phi_{0}=0 & \text { in } \Lambda_{T} \\ \Phi_{0}=0 & \text { on } \partial \Lambda_{T} \backslash \Omega_{T} \\ \Phi_{0}(x, T)=\varphi^{T}(x) & \text { on } \Omega_{T}\end{cases}
$$

By (4.6) and standard estimates, we have

$$
\left\|\tilde{\nabla} \Phi_{0}\right\|_{L^{2}\left(\Lambda_{T}\right)}^{2} \leq C M_{0}|\log \varepsilon|
$$

Step 2: "Gauge transformation" of $v_{\varepsilon}$. On $\Lambda_{T}$ we consider the map $w_{\varepsilon}: \Lambda_{T} \rightarrow \mathbb{C}$ defined by

$$
w_{\varepsilon}=v_{\varepsilon} \exp \left(-i \Phi_{0}\right) \quad \text { in } \Lambda_{T} .
$$

Note that $\left|w_{\varepsilon}\right|=\left|v_{\varepsilon}\right|$. The computations in Section 3 yield

$$
w_{\varepsilon} \times \delta w_{\varepsilon}=v_{\varepsilon} \times \delta v_{\varepsilon}-\left|v_{\varepsilon}\right|^{2} \delta \Phi_{0}=v_{\varepsilon} \times \delta v_{\varepsilon}-\delta \Phi_{0}+\left(1-\left|v_{\varepsilon}\right|^{2}\right) \delta \Phi_{0} \quad \text { in } \Lambda_{T}
$$

Since $\left|v_{\varepsilon}\right| \leq 1$ by assumption (4.1), we have

$$
\left|\nabla w_{\varepsilon}\right| \leq\left|\nabla v_{\varepsilon}\right|+\left|\nabla \Phi_{0}\right|
$$

hence it follows, from (4.7) and (2.19),

$$
\left\|\nabla w_{\varepsilon}\right\|_{L^{2}\left(\Lambda_{T}\right)}^{2}+\varepsilon^{-2} \|\left.\left(1-\left|w_{\varepsilon}\right|^{2}\right)\right|_{L^{2}\left(\Lambda_{T}\right)} ^{2} \leq C M_{0}|\log \varepsilon| .
$$

Note also that, since $\left|w_{\varepsilon}\right| \leq 1$ by $(4.1)$ and $\|\left.\left(1-\left|v_{\varepsilon}\right|^{2}\right)\right|_{L^{2}\left(\Lambda_{T}\right)} ^{2} \leq M_{0}(T+1) \varepsilon^{2}|\log \varepsilon|$, it follows by Hölder's inequality and (4.7) that, for $1 \leq p<2$,

$$
\left\|\left(1-\left|v_{\varepsilon}\right|^{2}\right) \delta \Phi_{0}\right\|_{L^{p}\left(\Lambda_{T}\right)}^{p} \leq C M_{0}(T+1) \varepsilon^{2-p}|\log \varepsilon|
$$

and similarly

$$
\left\|\left(1-\left|v_{\varepsilon}\right|^{2}\right) d \varphi^{T}\right\|_{L^{p}(\Omega)}^{p} \leq C M_{0} \varepsilon^{2-p}|\log \varepsilon|
$$


Next, we apply the HdR decomposition to $w_{\varepsilon}$ on $\Lambda_{T}$, so that

$$
w_{\varepsilon} \times d w_{\varepsilon}=\delta \Phi_{1}+\delta^{*} \Psi
$$

where $\Phi_{1}$ (resp. $\Psi$ ) is a function (resp. a 2-form) defined on $\Lambda_{T}$ such that

$$
\delta \Psi=0 \quad \text { in } \Lambda_{T}, \quad \Phi_{1}=0 \text { and } \Psi_{\top}=0 \text { on } \partial \Lambda_{T} .
$$

The equation for $\Psi$ is then $-\tilde{\Delta} \Psi=2 \tilde{J} w_{\varepsilon}$ in $\Lambda_{T}$, with boundary condition $\Psi_{\top}=0$ on $\partial \Lambda_{T}$, and

$$
\left(\delta^{*} \Psi\right)_{\top}=w_{\varepsilon} \times d w_{\varepsilon}=v_{\varepsilon} \times d v_{\varepsilon}-\left|v_{\varepsilon}\right|^{2} d \varphi^{T}=d^{*} \Psi^{T}+\left(1-\left|v_{\varepsilon}\right|^{2}\right) d \varphi^{T} \text { on } \Omega_{T} .
$$

In view of (4.6) and (4.11), we have for $1 \leq p<\frac{N+1}{N}<\frac{N}{N-1}$,

$$
\left\|\left(\delta^{*} \Psi\right) \top\right\|_{L^{p}\left(\Omega_{T}\right)} \leq C_{p}
$$

and hence, since $L^{p}\left(\Omega_{T}\right)=\left[L^{q}\left(\Omega_{T}\right)\right]^{*} \subset\left[W^{1-1 / q, q}\left(\Omega_{T}\right)\right]^{*}$, for $\frac{1}{p}+\frac{1}{q}=1$, it follows using the same arguments as in the proof of Proposition 3.1,

$$
\|\tilde{\nabla} \Psi\|_{L^{p}\left(\Lambda_{T}\right)} \leq C_{p}, \quad \forall 1 \leq p<\frac{N+1}{N}
$$

Finally, going back to (4.8), we have

$$
v_{\varepsilon} \times \delta v_{\varepsilon}=w_{\varepsilon} \times d w_{\varepsilon}-\delta \Phi_{0}+\left(1-\left|v_{\varepsilon}\right|^{2}\right) \delta \Phi_{0}=\delta\left(\Phi_{0}+\Phi_{1}\right)+\delta^{*} \Psi+\left(1-\left|v_{\varepsilon}\right|^{2}\right) \delta \Phi_{0} .
$$

We set

$$
\Phi=\Phi_{0}+\Phi_{1}, \quad \chi=\left(1-\left|v_{\varepsilon}\right|^{2}\right) \delta \Phi_{0},
$$

so that $v_{\varepsilon} \times \delta v_{\varepsilon}=\delta \Phi+\delta^{*} \Psi+\chi$. This completes the proof, in view of $(4.10,4.12)$ and (4.13).

\section{The Elliptic equation}

In this section, we turn to solutions $u \equiv u_{\varepsilon}$ of $(\mathrm{GL})_{\varepsilon}$ verifying (H1), (H2), (H3) or (H3bis). It follows from the analysis in Section 3.1, equations (3.4) and (3.6), that

$$
\int_{\Omega}|\nabla u|^{p} \leq C_{p}\left[\int_{\Omega}|\nabla \rho|^{p}+\int_{\Omega}|\nabla \varphi|^{p}+\int_{\Omega}|\nabla \psi|^{p}+\left(\varepsilon E_{\varepsilon}(u)\right)^{p / 2}\right]
$$

where $\rho=|u|$, and $\varphi \equiv \varphi_{\varepsilon}$ and $\psi \equiv \psi_{\varepsilon}$ are such that

$$
\begin{cases}u \times d u=d \varphi+d^{*} \psi & \text { in } \Omega \\ d \psi=0 & \text { in } \Omega \\ \varphi=0 & \psi_{\top}=0 \quad \text { on } \partial \Omega\end{cases}
$$

In order to prove Theorem 1 and Theorem 1bis it suffices, in view of (5.1), Remark 3.1 and Proposition 3.1, to bound $\varphi$ and $\rho$. 


\section{1. $\varphi$ vanishes!}

As a consequence of $(\mathrm{GL})_{\varepsilon}$, it turns out that $\varphi=0$. Indeed, taking the exterior product of $(\mathrm{GL})_{\varepsilon}$ by the solution $u$, we derive

$$
\operatorname{div}(u \times \nabla u)=u \times \Delta u=0 .
$$

In the formalism of differential forms, we may rewrite previous identity as $d^{*}(u \times d u)=0$. Since $u \times d u=d \varphi+d^{*} \psi$, it follows that $\varphi$ verifies

$$
\begin{cases}-\Delta \varphi=d^{*} d \varphi=0 & \text { in } \Omega \\ \varphi=0 & \text { on } \partial \Omega\end{cases}
$$

so that $\varphi=0$ in $\Omega$.

\section{2. $W^{1, p}$ estimates for $\rho$}

The equation for $\rho^{2}=|u|^{2}$ is

$$
-\Delta \rho^{2}+2|\nabla u|^{2}=\frac{2}{\varepsilon^{2}} \rho^{2}\left(1-\rho^{2}\right) .
$$

Proposition 5.1. Let $u$ be a solution of $(G L)_{\varepsilon}$ verifying (H1). Let $1 \leq p<2$. There exists some constant $K_{p}$ and $0<\alpha<1$ depending only on $p, M_{0}$ and $\Omega$, such that, for $0<\varepsilon<1$,

$$
\int_{\Omega}|\nabla \rho|^{p} \leq K_{p} \varepsilon^{\alpha}
$$

Proof. It is similar to the proof of ([11], Erratum). We introduce the set

$$
A=\left\{x \in \Omega, \rho(x)>1-\varepsilon^{1 / 2}\right\}
$$

and the function

$$
\bar{\rho}=\max \left\{\rho, 1-\varepsilon^{1 / 2}\right\},
$$

so that $\bar{\rho}=\rho$ on $A$ and $0 \leq 1-\bar{\rho} \leq \varepsilon^{1 / 2}$ in $\Omega$.

Next let $\zeta_{\varepsilon}$ be a function in $C_{c}^{\infty}(\Omega)$ such that $0 \leq \zeta_{\varepsilon} \leq 1$ on $\Omega, \zeta_{\varepsilon} \equiv 1$ on $\Omega_{\varepsilon} \equiv\left\{x \in \Omega\right.$, $\left.\operatorname{dist}(x, \partial \Omega) \geq \varepsilon^{1 / 2}\right\}$, and $\left|\nabla \zeta_{\varepsilon}\right| \leq C \varepsilon^{-1 / 2}$, where the constant $C$ depends only on $\Omega$.

Finally, we multiply equation (5.1) by $\zeta_{\varepsilon}\left(\bar{\rho}^{2}-1\right.$ ) (which is compactly supported in $\Omega$ ), and integrate over $\Omega$. We obtain

$$
\int_{\Omega} \nabla \rho^{2} \nabla \bar{\rho}^{2} \zeta_{\varepsilon}+\int_{\Omega} \frac{2 \rho\left(1-\rho^{2}\right)\left(1-\bar{\rho}^{2}\right)}{\varepsilon^{2}} \zeta_{\varepsilon}=\int_{\Omega}\left(1-\bar{\rho}^{2}\right)|\nabla u|^{2}+\int_{\Omega} \nabla \rho^{2} \nabla \zeta_{\varepsilon}\left(1-\bar{\rho}^{2}\right) .
$$

It follows that on the set $A_{\varepsilon}=\Omega_{\varepsilon} \cap A$ we have

$$
\begin{aligned}
\int_{A_{\varepsilon}}\left|\nabla \rho^{2}\right|^{2} & =\int_{A_{\varepsilon}} \nabla \rho^{2} \nabla \bar{\rho}^{2} \leq \varepsilon^{1 / 2} \int_{\Omega}|\nabla u|^{2}+\frac{2 C}{\varepsilon^{1 / 2}} \int_{\Omega}|\nabla \rho|\left|1-\rho^{2}\right| \\
& \leq \varepsilon^{1 / 2} \int_{\Omega}|\nabla u|^{2}+C(2 \varepsilon)^{1 / 2}\left[\int_{\Omega}|\nabla \rho|^{2}+\int_{\Omega} \frac{\left(1-\rho^{2}\right)^{2}}{2 \varepsilon^{2}}\right],
\end{aligned}
$$

hence, since $\rho \geq 1-\varepsilon^{1 / 2}$ on $A_{\varepsilon}$, we have, for $\varepsilon \leq 1 / 4$,

$$
\int_{A_{\varepsilon}}|\nabla \rho|^{2} \leq 4 \int_{A_{\varepsilon}}\left|\nabla \rho^{2}\right|^{2} \leq 16 C \varepsilon^{1 / 2} E_{\varepsilon}(u) \leq 16 C M_{0} \varepsilon^{1 / 2}|\log \varepsilon| .
$$


Set $W_{\varepsilon}=\Omega \backslash \Omega_{\varepsilon}, B=\Omega \backslash A$, so that

$$
\Omega=B \cup A_{\varepsilon} \cup W_{\varepsilon}
$$

From (H1) we deduce $\int_{B}\left(1-\rho^{2}\right)^{2} \leq 4 M_{0} \varepsilon^{2}|\log \varepsilon|$ and hence, since $(1-\rho) \geq \varepsilon^{1 / 2}$ on $B$, it follows $|B|$ $\leq 4 M_{0} \varepsilon|\log \varepsilon|$. Thus

$$
\int_{B}|\nabla \rho|^{p} \leq\left(\int_{\Omega}|\nabla \rho|^{2}\right)^{p / 2}|B|^{1-p / 2} \leq C|\log \varepsilon|^{p / 2}(\varepsilon|\log \varepsilon|)^{1-p / 2}
$$

i.e.

$$
\int_{B}|\nabla \rho|^{p} \leq C \varepsilon^{1-p / 2}|\log \varepsilon|
$$

Finally, we turn to $W_{\varepsilon}$. Clearly, by construction $\left|W_{\varepsilon}\right| \leq C \varepsilon^{1 / 2}$. Hence

$$
\int_{W_{\varepsilon}}|\nabla \rho|^{p} \leq\left(\int_{\Omega}|\nabla \rho|^{2}\right)^{p / 2}|W|^{1-p / 2} \leq C \varepsilon^{1 / 2-p / 4}|\log \varepsilon|^{p / 2} .
$$

Combining $(5.5)$ with $(5.4,5.6)$ and $(5.7)$ we derive the desired conclusion.

\subsection{Proof of Theorem 1 and Theorem 1 bis completed}

Combining the results of Proposition 5.1, Proposition 3.1, Remark 3.1, and the fact that $\varphi=0$, we deduce from (5.1) the conclusions of Theorem 1 and Theorem 1bis.

Remark 5.1. Let $\varepsilon_{n} \rightarrow 0$ be a subsequence such that $u_{\varepsilon_{n}} \rightarrow u_{*}$ in $W^{1, p}(\Omega)$ for every $1 \leq p<\frac{N}{N-1}, g_{\varepsilon_{n}} \rightarrow g_{*}$ in $H^{1 / 2}(\partial \Omega), \psi_{\varepsilon_{n}} \rightarrow \psi_{*}$ in $W^{1, p}\left(\Omega ; \Lambda^{2} \mathbb{R}^{N}\right)$. Passing to the limit in (5.2), we have

$$
u_{*} \times d u_{*}=d^{*} \psi_{*}
$$

We may also pass to the limit in the equation for $\psi_{\varepsilon}$, so that we are led to

$$
\begin{cases}-\Delta \psi_{*}=2 J u_{*} & \text { in } \Omega \\ \psi_{\top}=g_{*} \times d g_{*} & \text { on } \partial \Omega\end{cases}
$$

In particular, $\psi_{*}$, and hence $u_{*}$, belong to $C^{\infty}\left(\Omega \backslash \operatorname{supp}\left(J u_{*}\right)\right)$.

\section{The PARABOlic EQUation}

In this section, we turn to solutions $u \equiv u_{\varepsilon}$ of $(\mathrm{PGL})_{\varepsilon}$ verifying (H4), (H2), and (H3) or (H3bis). Applying Proposition 4.1 to $u$, we write

$$
u \times \delta u=\delta \Phi+\delta^{*} \Psi+\chi \quad \text { in } \Lambda_{T}
$$

where $\Phi$ is a function, $\chi$ a 1 -form, $\Psi$ a 2 -form defined on $\Lambda_{T}$, such that $\delta \Psi=0$ in $\Lambda_{T}, \Psi_{\top}=0$ on $\partial \Lambda_{T}$,

$$
\Phi=0 \quad \text { on } \Omega_{0} \cup \partial \Omega \times[0, T]
$$


and for $1 \leq p<\frac{N+1}{N}$, there are constants $C_{p}>0,0<\alpha_{p}<1$, such that

$$
\|\tilde{\nabla} \Psi\|_{L^{p}\left(\Lambda_{T}\right)} \leq C_{p}, \quad\|\chi\|_{L^{p}\left(\Lambda_{T}\right)} \leq C_{p} \varepsilon^{\alpha_{p}} .
$$

Moreover, we have, for $1 \leq p<2$,

$$
\int_{\Lambda_{T}}|\nabla u|^{p} \leq C_{p}\left(M_{0}(T+1)^{p / 2}+\int_{\Lambda_{T}}|\nabla \rho|^{p}+|\nabla \Phi|^{p}+|\tilde{\nabla} \Psi|^{p}+|\chi|^{p}\right),
$$

so that in order to prove Theorem 4 it suffices to bound $\rho$ and $\Phi$.

\section{1. $L^{p}$ estimates for $\nabla \rho$}

The equation for $\rho^{2}$ is

$$
\frac{\partial \rho^{2}}{\partial t}-\Delta \rho^{2}+2|\nabla u|^{2}=\frac{2}{\varepsilon^{2}} \rho^{2}\left(1-\rho^{2}\right) \quad \text { in } \Lambda_{T} .
$$

We then have:

Proposition 6.1. Let $u=u_{\varepsilon}$ be a solution of $(G L)_{\varepsilon}$ verifying (H4), (H2), and (H3) or (H3bis). Let $\rho=|u|$, and $1 \leq p<2$. There exists some constant $K_{p}>0$ and $0<\alpha<1$ such that, for every $0<\varepsilon<1$,

$$
\int_{\Lambda_{T}}|\nabla \rho|^{p} \leq K_{p} \varepsilon^{\alpha}
$$

Proof. It is similar to the proof of Proposition 5.1. One introduces $\bar{\rho}=\max \left\{\rho, 1-\varepsilon^{1 / 2}\right\}$ on $\Lambda_{T}$, and $\zeta_{\varepsilon}: \Omega \rightarrow \mathbb{R}^{+}$, as there. Multiplying (6.5) by $\bar{\rho} \zeta_{\varepsilon}$ on $\Lambda_{T}$ one obtains, computing as for (5.4),

$$
\int_{A_{\varepsilon}}\left|\nabla \rho^{2}\right|^{2} \leq \varepsilon^{1 / 2} \int_{\Lambda_{T}}|\nabla u|^{2}+C \varepsilon^{1 / 2} \int_{\Lambda_{T}}|\nabla \rho|^{2}+\frac{\left(1-\rho^{2}\right)^{2}}{2 \varepsilon^{2}}+\int_{\Lambda_{T}}\left|\frac{\partial \rho}{\partial t}(\bar{\rho}-1)\right|,
$$

where $A_{\varepsilon}=\left\{y \in \Lambda_{T}, \rho(y) \geq 1-\varepsilon^{1 / 2}\right\}$. The last term in inequality (6.6) can be bounded by $C \varepsilon \int_{\Lambda_{T}}|\tilde{\nabla} \rho|^{2}$ $+\frac{\left(1-\rho^{2}\right)^{2}}{4 \varepsilon^{2}}$. Hence

$$
\int_{A_{\varepsilon}}|\nabla \rho|^{2} \leq C M_{0}(T+1) \varepsilon^{1 / 2}|\log \varepsilon| .
$$

The proof is then completed as in the proof of Proposition 5.1.

\section{2. $\boldsymbol{L}^{p}$ estimates for $\boldsymbol{\nabla} \boldsymbol{\Phi}$}

Taking the exterior product of $(\mathrm{PGL})_{\varepsilon}$ with $u$, we are led to

$$
u \times \frac{\partial u}{\partial t}-\operatorname{div}(u \times \nabla u)=0 \quad \text { in } \Lambda_{T} .
$$

We have, in view of (6.1),

$$
\left\{\begin{array}{l}
u \times d u=d \Phi+\left(\delta^{*} \Psi+\chi\right)-P_{t}\left(\delta^{*} \Psi+\chi\right) d t \\
u \times \frac{\partial u}{\partial t}=\frac{\partial \Phi}{\partial t}+P_{t}\left(\delta^{*} \Psi+\chi\right) .
\end{array}\right.
$$


Here, for a 1 -form $\omega$ on $\Lambda_{T}$, we denote $P_{t}(\omega)$ its $d t$ component, i.e. if $\omega=\omega_{1} d x^{1}+\cdots+\omega_{N} d x^{N}+\omega_{t} d t$, then $P_{t}(\omega)=\omega_{t}$.

Combining (6.8) and (6.9) we are led to the equation for $\Phi$ :

$$
\frac{\partial \Phi}{\partial t}-\Delta \Phi=d^{*}\left(\delta^{*} \Psi+\chi-P_{t}\left(\delta^{*} \Psi+\chi\right) d t\right)-P_{t}\left(\delta^{*} \Psi+\chi\right) \quad \text { in } \Lambda_{T}
$$

Set $f=\left(f_{1}, \ldots, f_{N}\right)$, where

$$
f_{1} d x^{1}+\cdots+f_{N} d x^{N}=\delta^{*} \Psi+\chi-P_{t}\left(\delta^{*} \Psi+\chi\right) d t
$$

and let $g=-P_{t}\left(\delta^{*} \Psi+\chi\right)$. In view of (6.2) and (6.10) we obtain the following initial and boundary value parabolic problem for $\Phi$ :

$$
\left\{\begin{array}{llrl}
\frac{\partial \Phi}{\partial t}-\Delta \Phi & =\operatorname{div} f+g & \text { in } \Lambda_{T} & \\
\Phi(x, 0) & =0 & \forall x \in \Omega, \\
\Phi(x, t) & =0 & \forall x \in \partial \Omega, \forall t \in[0, T]
\end{array}\right.
$$

where, for every $1 \leq p<\frac{N+1}{N}$,

for some constant $C_{p}$ independent of $\varepsilon$.

$$
\|f\|_{L^{p}\left(\Lambda_{T} ; \mathbb{R}^{N}\right)}+\|g\|_{L^{p}\left(\Lambda_{T}\right)} \leq C_{p},
$$

Note that (6.11) is a well posed parabolic problem for $\Phi$. In view of standard parabolic estimates, we deduce

$$
\int_{\Lambda_{T}}|\nabla \Phi|^{p} \leq C_{p}(T) .
$$

\subsection{Proof of Theorem 4 completed}

Combining (6.3) with (6.12) and the result of Proposition 6.1, we deduce from (6.4) the conclusion of Theorem 4.

\subsection{Proof of Proposition 1}

Statement i) is straightforward. For statement ii) we argue as in Remark 5.1.

The first author is partially supported by European Grant ERB FMRX CT980201. The second author wishes to thank University Paris VI for the kind hospitality.

\section{REFERENCES}

[1] G. Alberti, S. Baldo and G. Orlandi, Variational convergence for functionals of Ginzburg-Landau type. Preprint (2001).

[2] L. Almeida, S. Baldo, F. Bethuel and G. Orlandi (in preparation).

[3] L. Almeida and F. Bethuel, Topological methods for the Ginzburg-Landau equation. J. Math. Pures Appl. 11 (1998) 1-49.

[4] L. Ambrosio and H.M. Soner, A measure theoretic approach to higher codimension mean curvature flow. Ann. Scuola Norm. Sup. Pisa Cl. Sci. (4) 25 (1997) 27-49.

[5] P. Baumann, C.-N. Chen, D. Phillips and P. Sternberg, Vortex annihilation in nonlinear heat flow for Ginzburg-Landau systems. Eur. J. Appl. Math. 6 (1995) 115-126.

[6] F. Bethuel, Variational methods for Ginzburg-Landau equations, in Calculus of Variations and Geometric evolution problems, Cetraro 1996, edited by S. Hildebrandt and M. Struwe. Springer (1999).

[7] F. Bethuel, J. Bourgain, H. Brezis and G. Orlandi, $W^{1, p}$ estimates for solutions to the Ginzburg-Landau equation with boundary data in $H^{1 / 2}$. C. R. Acad. Sci. Paris Sér. I Math. 333 (2001) 1069-1076.

[8] F. Bethuel, H. Brezis and F. Hélein, Asymptotics for the minimization of a Ginzburg-Landau functional. Calc. Var. Partial Differential Equations 1 (1993) 123-148. 
[9] F. Bethuel, H. Brezis and F. Hélein, Ginzburg-Landau Vortices. Birkhäuser, Boston (1994).

[10] F. Bethuel, H. Brezis and G. Orlandi, Small energy solutions to the Ginzburg-Landau equation. C. R. Acad. Sci. Paris Sér. I Math. 331 (2000) 763-770.

[11] F. Bethuel, H. Brezis and G. Orlandi, Asymptotics for the Ginzburg-Landau equation in arbitrary dimensions. J. Funct. Anal. 186 (2001) 432-520. Erratum (to appear).

[12] F. Bethuel and T. Rivière, Vortices for a variational problem related to superconductivity. Ann. Inst. H. Poincaré Anal. Non Linéaire 12 (1995) 243-303.

[13] J. Bourgain, H. Brezis and P. Mironescu, Lifting in Sobolev spaces. J. Anal. 80 (2000) 37-86.

[14] J. Bourgain, H. Brezis and P. Mironescu, On the structure of the Sobolev space $H^{1 / 2}$ with values into the circle. C. $R$. Acad. Sci. Paris Sér. I Math. 331 (2000) 119-124.

[15] H. Brezis and P. Mironescu, Sur une conjecture de E. De Giorgi relative à l'énergie de Ginzburg-Landau. C. R. Acad. Sci. Paris Sér. I Math. 319 (1994) 167-170.

[16] H. Federer, Geometric Measure Theory. Springer, Berlin (1969).

[17] Z.C. Han and I. Shafrir, Lower bounds for the energy of $S^{1}$-valued maps in perforated domains. J. Anal. Math. 66 (1995) 295-305.

[18] R. Hardt and F.H. Lin, Mappings minimizing the $L^{p}$-norm of the gradient. Comm. Pure Appl. Math. 40 (1987) 555-588.

[19] R. Jerrard, Lower bounds for generalized Ginzburg-Landau functionals. SIAM J. Math. Anal. 30 (1999) 721-746.

[20] R. Jerrard and H.M. Soner, Dynamics of Ginzburg-Landau vortices. Arch. Rational Mech. Anal. 142 (1998) 99-125.

[21] R. Jerrard and H.M. Soner, Scaling limits and regularity results for a class of Ginzburg-Landau systems. Ann. Inst. H. Poincaré Anal. Non Linéaire 16 (1999) 423-466.

[22] R. Jerrard and H.M. Soner, The Jacobian and the Ginzburg-Landau energy. Calc. Var. Partial Differential Equations (to appear).

[23] F.H. Lin, Some dynamical properties of Ginzburg-Landau vortices. Comm. Pure Appl. Math. 49 (1996) 323-359.

[24] F.H. Lin, Complex Ginzburg-Landau equations and dynamics of vortices, filaments, and codimension-2 submanifolds. Comm. Pure Appl. Math. 51 (1998) 385-441

[25] F.H. Lin, Rectifiability of defect measures, fundamental groups and density of Sobolev mappings, in Journées "Équations aux Dérivées Partielles", Saint-Jean-de-Monts, 1996, Exp. No. XII. École Polytechnique, Palaiseau (1996).

[26] F.H. Lin and T. Rivière, Complex Ginzburg-Landau equations in high dimensions and codimension two area minimizing currents. J. Eur. Math. Soc. 1 (1999) 237-311. Erratum, Ibid.

[27] F.H. Lin and T. Rivière, A quantization property for static Ginzburg-Landau vortices. Comm. Pure Appl. Math. 54 (2001) 206-228.

[28] F.H. Lin and T. Rivière, A quantization property for moving line vortices. Comm. Pure Appl. Math. 54 (2001) 826-850.

[29] L. Modica, The gradient theory of phase transitions and the minimal interface criterion. Arch. Rational Mech. Anal. 98 (1987) $123-142$.

[30] L. Modica and S. Mortola, Un esempio di Г-convergenza. Boll. Un. Mat. Ital. B 14 (1977) 285-299.

[31] T. Rivière, Line vortices in the U(1)-Higgs model. ESAIM: COCV 1 (1996) 77-167.

[32] T. Rivière, Dense subsets of $H^{1 / 2}\left(S^{2}, S^{1}\right)$. Ann. Global Anal. Geom. 18 (2000) 517-528.

[33] T. Rivière, Asymptotic analysis for the Ginzburg-Landau Equation. Boll. Un. Mat. Ital. $B 8$ (1999) 537-575.

[34] E. Sandier, Lower bounds for the energy of unit vector fields and applications. J. Funct. Anal. 152 (1997) 379-403; Erratum 171 (2000) 233.

[35] L. Simon, Lectures on Geometric Measure Theory, in Proc. of the Centre for Math. Analysis. Australian Nat. Univ., Canberra (1983).

[36] M. Struwe, On the asymptotic behavior of the Ginzburg-Landau model in 2 dimensions. J. Differential Equations 7 (1994) 1613-1624; Erratum 8 (1995) 224. 